\title{
Transmissible trimethoprim resistance in strains of Escherichia coli isolated from cases of infantile diarrhoea
}

\author{
A. LAMIKANRA, A. K. AKO-NAI* and J. B. OLA* \\ Faculties of Pharmacy and *Science, Obafemi Awolowo University, Ile-Ife, Nigeria
}

\begin{abstract}
Summary. Of 190 isolates of Escherichia coli from children aged up to 5 years with diarrhoea, $72(37.9 \%)$ were resistant to trimethoprim and of the 70 isolates tested, 38 transferred high level trimethoprim resistance $(\mathrm{MIC}>1000 \mathrm{mg} / \mathrm{L})$ into $E$. coli EC 1005. Of the enterotoxigenic isolates from which trimethoprim resistance was transferred, $41 \cdot 2 \%$ also contained transmissible toxigenic characteristics. All the trimethoprim-resistant isolates were resistant to other antibiotics.
\end{abstract}

\section{Introduction}

Diarrhoeal disease is responsible for a large proportion of deaths in children under the age of 2 years in developing countries (Snyder and Merson, 1982). One cause of this diarrhoea, Escherichia coli, is part of the normal gut flora and is, therefore, more likely to be transferred between children. $E$. coli can also spread from the gut to infect other parts of the body such as the urinary tract. These infections are usually treated with anti-infective agents, one of the most frequently used being trimethoprim, singly or in combination with sulphamethoxazole. Therefore, the sensitivity of $E$. coli strains to trimethoprim has been studied by many workers, including Murray et al. $(1982,1985)$ and Huovinen (1984).

A high incidence of resistance to an antibiotic within a given population has long been accepted as evidence of widespread use of that antibiotic. The converse, that in the absence of widespread use of an antibiotic the resistance rate is low, should also be true. However, this is not always the case because, as shown by Sköld et al. (1986), people who have never been exposed to a particular antibiotic may nevertheless harbour organisms that are resistant to that antibiotic. This is made possible by the transfer of linked plasmid- and transposonmediated antibiotic resistances which have been acquired through cross-infection of the antibioticinnocent host and transfer of resistance to enteric organisms in that host. Transmissible plasmids are

Received 8 Dec. 1988; accepted with modifications 28 Dec. 1989. particularly likely to transfer in this way and the presence of such plasmids is thus epidemiologically significant. The spread of antibiotic resistances in this manner is also enhanced (Murray et al., 1985) by poor personal and social hygiene, overcrowding and other conditions which encourage the frequent exchange of enteric organisms. Such conditions prevail in the environment studied.

\section{Materials and methods}

The organisms used in this study were isolated from children aged up to 5 years in whom the diagnosis of diarrhoea was made by a physician on the basis of frequent watery stools (usually more than four a day) with mucus or blood in the stool, abdominal pain and signs of dehydration. Samples were collected from all the children when they attended the Oral Rehydration Therapy (ORT) outpatient clinic at the Oni Memorial Hospital in Ibadan, a city with more than 1 million inhabitants.

\section{Samples and isolation of $E$. coli}

Faecal samples were collected from the patients into universal sterile disposable containers (Sterilin Ltd, Feltham) and on arrival in the laboratory were immediately inoculated on Eosin Methylene Blue (EMB), MacConkey and Blood Agar plates (all media were from Difco Laboratories). All plates were incubated aerobically at $37^{\circ} \mathrm{C}$ for $24 \mathrm{~h}$ or until visible colonies appeared. Lactose-fermenting colonies on EMB and MacConkey agar plates were picked with a sterile inoculating needle and examined first by Gram's stain. These lactosefermenting colonies were often haemolytic on blood agar and were confirmed as E. coli by the API IOS system (API Laboratory Products, La Balm Les Grottes, 38290 Montalieu-Vercieu, France). Enteropathogenic E. coli 
(EPEC) strains were identified by slide agglutination with polyvalent and monovalent $E$. coli sera (Wellcome Laboratories, Beckenham). Enterotoxigenic $E$. coli (ETEC) strains were identified by staphylococcal coagglutination for the detection of heat-labile enterotoxin (LT) with commercially available kits (Pharmacia Diagnostics, Uppsala, Sweden) (Ronnberg and Wadstronı, 1983).

\section{Antibiotic sensitivity testing}

Antibiotic sensitivities were tested by disk diffusion on IsoSensitest Agar (Oxoid) with antibiotic disks from AB Biodisk (Solna, Sweden) containing ampicillin $10 \mu \mathrm{g}$, cephalothin $30 \mu \mathrm{g}$, trimethoprim $1.2 \mu \mathrm{g}$, sulphamethoxazole $23.8 \mu \mathrm{g}$, streptomycin $30 \mu \mathrm{g}$, chloramphenicol $30 \mu \mathrm{g}$, tetracycline $30 \mu \mathrm{g}$, gentamicin $30 \mu \mathrm{g}$, spectinomycin $30 \mu \mathrm{g}$ and nalidixic acid $30 \mu \mathrm{g}$. The inocula were spread on the surface of dry plates with sterile cotton swabs which had been dipped in a diluted suspension of the organisms in sterile normal saline. The plates were incubated at $37^{\circ} \mathrm{C}$ for $30 \mathrm{~min}$ before the disks were applied and then refrigerated at $4^{\circ} \mathrm{C}$ for $30 \mathrm{~min}$ before incubation at $37^{\circ}$ for $18 \mathrm{~h}$, after which the zones of inhibition were measured. Proteus mirabilis ATCC 14273, Staphylococcus aureus ATCC A25923 and Enterobacter aerogenes ATCC 13048 (American Type Collection, Richville, MD, USA) were used as controls. Determination of resistance was according to the manufacturer's instructions.

\section{Determination of trimethoprim MIC}

Of the $E$. coli strains, 72 were found to be resistant to trimethoprim by the disk diffusion method and the MIC was estimated in each case by the agar dilution method (Ericsson and Sherris, 1971). The organisms were grown in IsoSensitest Broth (Oxoid) at $37^{\circ} \mathrm{C}$ for $18 \mathrm{~h}$ and a 1 in 1000 dilution of the bacterial suspension was made to give $c .1 \cdot 0 \times 10^{5} \mathrm{cfu} / \mathrm{ml}$. The organisms were then transferred on to the surface of dried IsoSensitest Agar plates containing doubling concentrations $(12 \cdot 5-1600 \mathrm{mg} / \mathrm{L})$ of trimethoprim with a multipoint inoculator (Denley, Billingshurst, Sussex) which delivered c. $1 \mu \mathrm{l}$ of the suspension. The plates were incubated at $37^{\circ} \mathrm{C}$ for $24 \mathrm{~h}$. The lowest concentration inhibitory to bacterial growth was taken as the MIC.

\section{In-vitro transconjugation of trimethoprim resistance}

In $E$. coli both trimethoprim resistance and toxigenicity are plasmid-borne. The incidence of transmissible plasmids mediating these properties in this study population was thus thought to be of considerable interest.

Cultures of trimethoprim-resistant $E$. coli were grown overnight in IsoSensitest Broth and $0.4 \mathrm{ml}$ of the bacterial suspension was placed on filter paper with an equal volume of a similarly prepared culture of $E$. coli $\mathrm{EC} 1005$ $\mathrm{Na}^{\mathrm{r}}, \mathrm{Tm}^{\mathrm{s}}$, met. The filter paper with the mixed culture was placed on IsoSensitest agar and incubated at $37^{\circ} \mathrm{C}$ for $24 \mathrm{~h}$. The growth was harvested with $3 \mathrm{ml}$ of sterile normal saline and the thick suspension obtained was streaked on over-dried IsoSensitest agar plates containing nalidixic acid $40 \mathrm{mg} / \mathrm{L}$ plus trimethoprim $10 \mathrm{mg} / \mathrm{L}$ to select trimethoprim-resistant transconjugants. The colonies obtained after growth at $37^{\circ} \mathrm{C}$ for $24 \mathrm{~h}$ were restreaked on fresh selecting plates and incubated at $37^{\circ}$ for $24 \mathrm{~h}$. Colonies on these plates were then tested for the methionine marker of strain EC 1005. The trimethoprimresistant transconjugants obtained were also tested for their sensitivity to all the antibiotics to which the donor organisms were resistant as well as their ability to produce heat-labile toxins as earlier described.

\section{Results}

\section{Isolation of E. coli}

Of the 247 different enterobacterial isolates obtained in this study, $100(40.5 \%)$ were enteropathogenic $E$. coli strains, $62(25 \cdot 1 \%)$ were enterotoxigenic $E$. coli strains and there were $28(11 \cdot 3 \%)$ other $E$. coli strains-in all $190(76.9 \%) E$. coli strains. The other organisms isolated were Proteus spp. 2 (0.8\%), Klebsiella spp. 11 (4.5\%) Enterobacter spp. $13(5 \cdot 3 \%)$, Salmonella spp. $13(5 \cdot 3 \%)$, Shigella spp. $7(2.8 \%)$, Serratia marcescens $4(1.6 \%)$, Citrobacter freundii $4(1.6 \%)$ and Hafnia alvei $4(1.6 \%)$.

\section{Antibiotic sensitivity testing}

The antibiotic sensitivity patterns are shown in table I.

\section{Determination of trimethoprim MIC}

Thirty-five (35\%) EPEC, $28(45 \cdot 2 \%)$ ETEC and $9(32 \cdot 1 \%)$ other E. coli strains were resistant to trimethoprim-an overall figure of $72(37.9 \%)$ for all $E$. coli strains. Trimethoprim resistance in these isolates was quantified by MIC determination; 18 $(51.4 \%)$ of the EPEC strains, $21(75 \%)$ of the ETEC strains, and $3(33.3 \%)$ other E. coli strains were classified as highly resistant. All the other isolates had MIC values below $1000 \mathrm{mg} / \mathrm{L}$ (table II).

\section{Transconjugation of trimethoprim resistance}

Of the $70 \mathrm{E}$. coli isolates tested for the transconjugation of trimethoprim resistance, $38(54 \cdot 2 \%)$ were able to transfer resistance to $E$. coli $\mathrm{EC} 1005-$ $18(54.5 \%)$ of the EPEC strains, $17(60.4 \%)$ of the ETEC strains but only $3(33 \%)$ of the other E. coli strains. All the organisms from which trimethoprim resistance was transferred were highly resistant, i.e., the MIC of trimethoprim for them exceeded $1000 \mathrm{mg} / \mathrm{L}$. 
Table I. Incidence of antibiotic resistances amongst $E$. coli strains isolated from children with diarrhoea

\begin{tabular}{lccrr}
\hline & \multicolumn{4}{c}{ Number $(\%)$ of resistant isolates } \\
\cline { 2 - 5 } Antibiotic & EPEC & ETEC & E. coli & Total \\
\hline Tetracycline & $88(88 \cdot 0)$ & $58(93 \cdot 5)$ & $26(92 \cdot 9)$ & $172(90 \cdot 5)$ \\
Gentamicin & $85(85 \cdot 0)$ & $50(80 \cdot 6)$ & $21(75 \cdot 0)$ & $156(82 \cdot 1)$ \\
Trimethoprim & $35(35 \cdot 0)$ & $28(45 \cdot 2)$ & $9(32 \cdot 1)$ & $72(37 \cdot 9)$ \\
Sulphamethoxazole & $81(81 \cdot 0)$ & $56(90 \cdot 3)$ & $25(89 \cdot 3)$ & $162(85 \cdot 3)$ \\
Spectinomycin & $73(73 \cdot 0)$ & $51(82 \cdot 3)$ & $24(85 \cdot 7)$ & $148(77 \cdot 9)$ \\
Ampicillin & $58(58 \cdot 0)$ & $41(66 \cdot 1)$ & $17(60 \cdot 7)$ & $116(60 \cdot 4)$ \\
Cephalothin & $47(47 \cdot 0)$ & $35(56 \cdot 5)$ & $11(39 \cdot 3)$ & $93(48 \cdot 9)$ \\
Chloramphenicol & $70(70 \cdot 0)$ & $44(70 \cdot 9)$ & $19(67 \cdot 9)$ & $133(70 \cdot 0)$ \\
Streptomycin & $63(63 \cdot 0)$ & $47(75 \cdot 8)$ & $15(53 \cdot 6)$ & $125(65 \cdot 8)$ \\
Nalidixic acid & $4(4 \cdot 0)$ & $4(6 \cdot 5)$ & $2(7 \cdot 1)$ & $10(5 \cdot 3)$ \\
& & & & \\
\hline
\end{tabular}

Transconjugation of toxigenicity and trimethoprim resistance

Of the 17 ETEC isolates with transferable trimethoprim resistance, $7(40 \cdot 1 \%)$ were also shown to harbour transmissible heat-labile toxigenicity.

\section{Co-transfer of other antibiotic resistance markers with trimethoprim resistance}

Many of the transconjugants obtained in this study were resistant to at least some of the other antibiotics to which their donor organisms were resistant, presumably because they were transferred on the same plasmid as trimethoprim resistance, or were transferred on plasmids which were mobilised by the plasmid which coded for trimethoprim resistance (or vice versa). This is of epidemiological importance for the dissemination of antibiotic resistance within the community. In this study the highest rate of co-transfer was $70.3 \%$ for gentamicin, followed by $63.2 \%$ and $59.4 \%$ for sulphamethoxazole and chloramphenicol respectively. The co-transfer rate for streptomycin was $55.9 \%$, for ampicillin $48.5 \%$ and for cephalothin $45.5 \%$. Noticeably lower figures of $27.8 \%$ and $21.5 \%$ were obtained for spectinomycin and tetracycline respectively.

\section{Discussion}

Our results show that trimethoprim resistance was transferable in $54.5 \%$ of the resistant $E$. coli strains. This is considerably higher than incidences which have been recorded in several similar studies but is similar to that observed by Young et al. (1986) and by Lamikanra and Ndep (1989) in trimethoprim-resistant urinary tract pathogens isolated from patients in India and Nigeria respectively. This high incidence of transferability suggests that the high incidence of trimethoprim resistance encountered in this study is at least in part due to the spread of resistance plasmids through the community. The high frequency of transfer is also related to the fact that up to $60 \%$ of the trimethoprim-resistant organisms were highly resistant to the drug and, as reported by Brumfitt et al. (1977), Amyes et al. (1978), Datta et al. (1980) and Young et al. (1986), such high level trimethoprim resistance is transferred much more frequently than low level trimethoprim resistance.

Table II. MICs of trimethoprim for trimethoprim-resistant isolates of $E$. coli

\begin{tabular}{lccccc}
\hline & \multicolumn{5}{c}{ Number of isolates with MIC value (mg/L) } \\
\cline { 2 - 6 } Organisms & 1000 & 500 & 250 & 100 & $<100$ \\
\hline EPEC & 18 & 8 & 6 & 2 & 1 \\
ETEC & 20 & 3 & 3 & 2 & 0 \\
E. coli (other) & 3 & 3 & 1 & 1 & 1 \\
Total (\%) & $41(56.9)$ & $14(19.4)$ & $10(13.9)$ & $5(16.9)$ & $2(2.8)$ \\
\hline
\end{tabular}


Toxigenicity in $E$. coli is also plasmid-borne and results from this study showed that $7(41.2 \%)$ of the 17 ETEC isolates from which trimethoprim was transferred also contained transmissible toxigenicity factors. It should also be noted that only heat labile toxigenicity was investigated in this study, thus, the figure reported here is probably an underestimate of the incidence of transferable toxigenicity in the sample. The significance of this is that toxigenic strains may be able to spread through the agency of the trimethoprim resistance plasmid. Therefore, the incidence of toxigenic $E$. coli would increase as the use of trimethoprim increases within the community. Conversely, antibiotic resistance which is co-transferable with toxigenicity would also be disseminated by toxigenic $E$. coli. This mechanism for the spread of antibiotic resistances must be seriously considered in a population such as ours, in which $E$. coli strains feature prominently in infantile diarrhoea.

Our results show a very high rate of resistance to many antibiotics, an indication that effective antibiotic therapy in this population is now in

\section{REFERENCES}

Amyes S G B, Emmerson A M, Smith J T 1978 R-factor mediated trimethoprim resistance: result of two threemonth clinical surveys. Journal of Clinical Pathology 31 : 850-854.

Brumfitt W, Hamilton-Miller J M T, Grey D 1977 Trimethoprimresistant coliforms. Lancet 2: 926.

Datta $N$ et al. 1980 Distribution of genes for trimethoprim and gentamicin resistance in bacteria and their plasmids in a general hospital. Journal of General Microbiology 118: 495508 .

Ericsson H M, Sherris J C 1971 Antibiotic sensitivity testing: agar dilution method. Acta Pathologica et Microbiologica Scandinavica 217 Suppl B: 67-68.

Huovinen P 1984 Prevalence of trimethoprim resistance among bacteria isolated from urinary tract indicating characterization of transferable resistance. Thesis, University of Turku, Finland.

Lamikanra A, Ndep R B 1989 Trimethoprim resistance in urinary tract pathogens in two Nigerian hospitals. Journal of Antimicrobial Chemotherapy 23: 151-154.

Murray B E, Rensimer E R, Dupont H L 1982 Emergence of high-level trimethoprim resistance in faecal Escherichia coli jeopardy. The widespread resistance encountered with gentamicin is of special interest. In an earlier study of 244 urinary tract pathogens, $40.6 \%$ of which were $E$. coli, in a comparable community (Lamikanra and Ndep, 1989), the incidence of gentamicin resistance was only $13.8 \%$, compared with the $82.1 \%$ observed in the present study. Moreover, gentamicin, which is available only in injectable form, does not lend itself to the type of abuse that is common with orally administered antibiotics. The high resistance rate to gentamicin in this study is as worrisome as it is unexpected. If the antibiotics to which gentamicin resistance is linked are, as trimethoprim, widely used in the community, then gentamicin resistance is likely to spread through the community as has been the case in our study group. Support for the inference that gentamicin resistance in the group has spread in conjunction with the resistance to other antibiotics is provided by the fact that the rate of co-transfer of gentamicin and trimethoprim resistances in this study was $70.3 \%$. Similar co-transfer with other antibiotics is a distinct possibility in these isolates.

during oral administration of trimethoprim or trimethoprim-sulphamathoxazole. New England Journal of Medicine 306: $130-135$.

Murray B E et al. 1985 Increasing resistance to trimethoprimsulphamethoxazole among isolates of Escherichia coli in developing countries. Journal of Infectious Diseases 152: 1107-1113.

Ronnberg B, Wadstrom T 1983 Rapid detection by a coagglutination test of heat-labile enterotoxin in cell lysates from blood agar-grown Escherichia coli. Journal of Clinical Microbiology 17: 1021-1025.

Sköld O, Boethius G, Steen R 1986 Correlation of drug untilization data for trimethoprim in a defined population with patterns of resistance among bacteria causing urinary tract infections. Scandinavian Journal of Infectious Diseases 18: 451-455.

Snyder J D, Merson M H 1982 The magnitude of the global problem of acute diarrheal disease: a review of active surveillance data. Bulletin of the World Health Organization 60: 605-613.

Young H-K, Jesudason M V, Koshi G, Amyes S G B 1986 Trimethoprim resistance amongst urinary pathogens in south India. Journal of Antimicrobial Chemotherapy 17: 615621 . 